RAEIC, Revista de la Asociación Española de Investigación de la Comunicación

\title{
MasterChef Junior: el público infantil como producto de entretenimiento
}

\section{MasterChef Junior: children as entertainment}

Fuentes-Rosquete, Ariana

Universidad Carlos III de Madrid (UC3M)

100386196@alumnos.uc3m.es

González-González, Adriana

Universidad Carlos III de Madrid (UC3M)

100386318@alumnos.uc3m.es

Hernández-Chávez, Patricia

Universidad Carlos III de Madrid (UC3M)

100386166@alumnos.uc3m.es

Forma de citar este artículo:

Fuentes-Rosquete, A., González-González, A., y Hernández-Chávez, P. (2020). “MasterChef Junior: el público infantil como producto de entretenimiento", RAEIC, Revista de la Asociación Española de Investigación de la Comunicación, vol. 7, núm. 13, 272-289.

https://doi.org/10.24137/raeic.7.13.13

\section{Resumen:}

Este artículo tiene por objeto analizar el programa de cocina MasterChef Junior con el fin de conocer si sus contenidos enfatizan en la educación de los menores o, si por el

RAEIC, Revista de la Asociación Española de Investigación de la Comunicación vol. 7, núm. 13 (2020), 272-289 
contrario, se dirigen indirectamente a un público adulto y responden a intereses de anunciantes en una televisión pública que no admite publicidad. El estudio parte de un análisis de discurso de episodios de la quinta temporada y de contenido de los tweets relacionados con el programa. Concretamente desde el 20 de diciembre de 2017 al 10 de enero de 2018 y se realizó sobre una muestra de 150 tuits publicados.

Palabras clave: público infantil, MasterChef Junior, industria del entretenimiento, rating, intereses comerciales, prime time.

\section{Abstract:}

This article has as its main goal to analyze the cooking show MasterChef Junior with the aim of knowing exactly if its content emphasizes the education of the children or, if otherwise, the focus, indirectly, is a mature audience and if it responds to the interests of the advertisers in a public television that doesn't admit ads. The study starts with an analysis of discourse of some episodes of the fifth season and an analysis of content of the tweets related to the program. Specifically from the 20th December of 2017 to the 10th January of 2018 and over a sample of 150 tweets posted.

Keywords: young audience, MasterChef Junior, entertainment industry, rating, commercial interests, prime time.

\section{INTRODUCCIÓN}

Este proyecto se orienta a analizar 'El público infantil como producto de entretenimiento'. Se centrará en el caso concreto de MasterChef Junior (Shine Iberia, 2013-), considerado un programa orientado a un público infantil. Por tanto, el objeto de estudio se centrará en la utilización del público infantil en programas televisivos de la industria del entretenimiento. Se abordarán varias aristas como, por ejemplo, la influencia de las redes sociales en la promoción de este tipo de programaciones o los intereses económicos existentes alrededor de este. 
MasterChef Junior es un programa de televisión gastronómico emitido en La 1 de Televisión Española (TVE), y cuyo objetivo es encontrar al mejor cocinero amateur infantil de España. Está presentado por Eva González y cuenta con los chefs Jordi Cruz, Samantha Vallejo y Pepe Rodríguez como jueces de los concursantes. El concurso comenzó a emitirse en diciembre de 2013, y acumula seis ediciones hasta la fecha. La edad media de los niños que participan es de 11 años. Además, la media de la cuota de pantalla de esta edición objeto de análisis fue del 16,7\% (según datos de datos de Kantar Media con invitados).

El programa no incluye publicidad como tal, ya que RTVE no cuenta con esta vía de financiación desde la entrada en vigor de la Ley 8/2009, del 28 de agosto, de financiación de la Corporación de Radio y Televisión Española. Esta ley ha traído consigo numerosas críticas, opiniones y observaciones, así como algunas denuncias por supuesto incumplimiento de la ley (Gallardo, Elías y Jiménez-Marín, 2018). RTVE ha ido incorporando espacios televisivos apoyados con fórmulas que la industria publicitaria y televisiva recogen bajo distintas etiquetas, entre ellas: patrocinio deportivo o cultural y Branded content. La primera imposibilita a TVE aplicar esos posibles patrocinios a cualquier otro programa que no sea deportivo o cultural. Pero como todas las etiquetas, barreras y clasificaciones, sus límites son confusos (Segarra, Rodríguez e Hidalgo, 2014). Por lo tanto, para referirnos a la presencia de marcas o de publicidad en el formato, utilizamos el término "patrocinio cultural".

Mediante análisis de contenido y de discurso al programa y a las redes sociales de los concursantes se ha pretendido contabilizar con qué frecuencia, bajo qué condiciones y con qué intenciones se manifiestan estos patrocinios. A su vez, se ha tratado de analizar con qué propósitos son utilizados los niños en el programa Masterchef Junior, desde una perspectiva no examinada con anterioridad. Las investigaciones actuales sobre este programa se limitan a determinar si su emisión es capaz de cambiar los hábitos alimenticios de los espectadores, por ejemplo, con recetas sanas, equilibradas, o incluso con consejos y explicaciones que dan los presentadores o los propios concursantes acerca de estos hábitos (Aguilar, 2015). Sin embargo, este estudio pretende analizar a 
qué tipo de audiencia va destinado tanto el programa como el patrocinio cultural que se emite durante el mismo.

\section{MASTERCHEF JUNIOR, ENTRE LA EDUCACIÓN Y EL INTERÉS COMERCIAL}

Para Desde los inicios de la televisión, España ha trasladado a las audiencias el interés gastronómico por medio de diversos formatos como Mesa y mantel (TVE 1, 1958) o Masterchef (TVE 1, 2013). Sin embargo, en la actualidad se vive un cambio de paradigma en el cual este tipo de programas televisivos ya no tratan de aproximar la profesionalización de la cocina a la sociedad, sino que «desplazan el mensaje esencial y la finalidad del programa de cocina instructivo a una intención mucho más expectativa [...] lo importante es distraerse, evadirse y no tanto recibir la formación culinaria» (Rodríguez, Hidalgo y Segarra, 2014, p.149). Por ello, no es de extrañar que los niños adquieran un rol activo y protagónico, emprendiendo actividades y tareas en donde se exhiben como capaces de iniciativa creativa y de resolución inteligente de problemas (Fuenzalida, 2008, p.50).

A finales de los años 90 se comienza a construir una imagen distinta acerca del niño que influirá en su relación con la televisión y con la elaboración de programas (Fuenzalida, 2017a, p.58). De ahí que surjan canales para un público infantil centrados en intereses comerciales que exhiben programas con un rating atractivo para los anunciantes de publicidad de productos para niños, así como los intereses de los productores de televisión infantil que buscan vender sus programas para financiar nuevas producciones (Fuenzalida, 2017b, p.47).

Se ha hecho especial hincapié en la introducción de los medios de comunicación a los sistemas educativos para que los alumnos sean capaces de realizar análisis críticos de los mensajes recibidos, así como descubrir su posición dentro de los medios (Marín, 2006, p.195). Por ello, esta situación reclama una intervención de los mass media en el ámbito educativo. Para Ballesta $(2002$, p.2) la educación en medios debe permitir al alumno entender y comprender la información que recibe a través de dichos canales comunicativos. 
El aumento de la publicidad indirecta y encubierta en los programas para niños ha incrementado en los últimos años. Junto con esta presencia excesiva, se ha señalado que muchos de los anuncios que son emitidos en el espacio de programación infantil son inadecuados para las edades en las que se mueven estos formatos (Digón, 2008, p.68). Asimismo, los valores que se transmiten por medio de la publicidad introducida en estos espacios intensifican para Alonso, Matilla y Vázquez (1995), ideas relacionadas con el individualismo, el deseo de tener para ser feliz, la competitividad, el conseguir cosas sin esfuerzo, el sexismo, etc.

A su vez, un estudio promovido por el Instituto Oficial de Radio y Televisión y cuyo informe salió a la luz en 2005, desveló lo que deberían ser contenidos prioritarios de los programas infantiles como educar en el respeto a la persona y los grupos sociales; fomentar y facilitar la convivencia, estimular la tolerancia, propugnar la igualdad en todas sus acepciones (sexos, razas, creencias, oportunidades...), evitando la discriminación de género que adjudica determinados valores a las niñas y otros muy distintos a los niños; apoyar la integración; promover la no violencia; potenciar la no discriminación (Río y Román, 2005, p.27).

A pesar de que la televisión no enseña nada por sí sola ya que los niños necesitan la conexión de esos mensajes con sus experiencias y pensamientos previos, se puede corroborar que en la llamada sociedad de la información impera la cultura de la imagen y que el medio televisivo impone sus normas y modelos de comunicación, llevando a cabo una importante influencia en la transmisión de la ideología dominante (Giordano, 2000, p.30). Dicha ideología dominante, basada en la implantación de un modelo de sociedad neoliberal cuyos principales beneficiarios son las grandes empresas, es difundida de manera eficaz a través del medio televisivo.

Por ello, el objetivo principal que se quiere abordar con esta investigación es el uso que se lleva a cabo de los niños y de su inocencia para publicitar no solo el programa, sino marcas (como El Corte Inglés, Zara, Bosch...), o incluso productos propios de MasterChef Junior como cursos de cocina, campamentos de verano para niños o merchandising. Como apunta Carlos Soria (1999, p.154), la verdad y, en especial la persuasión 
publicitaria, presentan un mayor número de problemas cuando sus receptores aún poseen esa ingenuidad e inmadurez de los primeros años.

La tecnología, y en especial, las redes sociales, se han ido desarrollando en los últimos años de una manera inimaginable. El Eurobarómetro 2005/06 muestra que más de cuatro de cada cinco jóvenes de 12-17 años y uno de cada dos de 8-9 años han utilizado internet (Mediappro, 2006). El problema del riesgo de daños a la seguridad y el desarrollo social de los niños es una preocupación que ha ido incrementando a medida que los propios contenidos y servicios en la Red se expanden y crecen (Livingstone, 2007, p.52). Los niños y los jóvenes suelen situarse a la vanguardia de las nuevas actividades antes de que se lleve a cabo la verificación por parte de los adultos y la intervención reguladora, lo que supone una exposición ante experiencias negativas que no han sido previstas, para las cuales pueden no estar preparados (Livingstone, 2007).

En este proyecto de investigación se pretenderá indagar acerca del verdadero fin de la utilización de los niños en las redes sociales y ver si realmente se lleva a cabo por entretenimiento o hay fines comerciales detrás. De igual manera, se quiere evaluar el papel que los participantes desempeñan dentro del formato como colaboradores activos de la industria del entretenimiento. De esta forma, el trabajo pretende tener como punto de referencia los estudios emitidos en torno a la publicidad y a la audiencia hacia la que van dirigidos estos programas, aplicándolos a MasterChef Junior y tratando de solventar aquellos posibles vacíos que existan relacionados con esta materia.

\section{METODOLOGÍA}

Con el objeto de estudio y los objetivos principales ya definidos, se han planteado varias hipótesis que se esperan que, al final de esta investigación, se puedan llegar a confirmar, a partir de las metodologías utilizadas. En primer lugar, las cuentas de Twitter de los concursantes de MasterChef Junior son utilizadas como medio publicitario del programa y no simplemente como una fuente de comunicación entre los participantes y la audiencia, como se pretende hacer ver. En segundo lugar, según lo que se ha observado y según algunos artículos leídos, los concursantes manifiestan actitudes que no se 
corresponden con lo que supuestamente se quiere inculcar al público infantil. Se trata de un programa duro que puede hacer que los niños lleguen a situaciones de gran estrés.

La investigación se centra en la quinta edición de MasterChef Junior, que tuvo lugar entre 2017 y 2018, debido a que es la temporada que más telespectadores consiguió reunir. En la exploración participan siete de los concursantes de dicha edición, niños de entre 9 y 12 años, tres niños (Juan Antonio, 10 años; Gonzalo y Héctor, 12 años) y cuatro niñas (Esther, 10 años; Lucía, 12 años; María y María Arias, 9 años), todos semifinalistas del programa.

Para la selección de la muestra se tomó en cuenta la relevancia mediática de los aspirantes, así como el haber llegado hasta una fase avanzada dentro del formato televisivo. Los niños y niñas escogidos cumplen el criterio de ser semifinalistas de MasterChef Junior y haber adoptado un papel protagonista y participativo tanto en el desarrollo de las pruebas como en los totales, que funcionan como un recurso adicional y complementario al formato. En particular, se han elegido niños mediáticos: niños que publican y que poseen cuentas de Twitter y de Instagram, e incluso que comparten vídeos propios de manera pública en la plataforma Youtube.

El trabajo de campo se desarrolló entre mediados de marzo y principios de mayo de 2019, llevando a cabo un profundo análisis de discurso de la quinta temporada de MasterChef Junior, en especial, los dos últimos capítulos emitidos (semifinal y final). Dentro de cada episodio se analizan en profundidad los fragmentos en los que los participantes realizan comentarios y manifiestan valores o características relacionadas con su manera de ser o con el papel que han adquirido debido a la exposición televisiva. Además, se ha tenido muy en cuenta la fuerte labor publicitaria que se exhibe dentro del programa a pesar de emitirse en un canal (RTVE) que ha eliminado los anuncios. También se han tomado en cuenta las redes sociales de cada uno de los participantes (Twitter) como un modo de difusión y de publicidad para el programa.

La segunda técnica empleada ha sido un análisis de contenido, tomando como base para ello la red social Twitter, más concretamente los perfiles de los finalistas y semifinalistas de la quinta edición de MasterChef Junior. De entre los siete concursantes de la semifinal 
se han escogido a cinco, basándose en su relevancia mediática, número de Tweets, interacción con el programa y en la interacción con sus compañeros. Los cinco escogidos son Esther, María Arias, Juan Antonio, Gonzalo y Lucía, componiendo un total de tres niñas y dos niños, tres finalistas y dos semifinalistas. En el análisis se han examinado treinta tweets de cada concursante. En ellos se ha tenido en cuenta la intención del mensaje, su formato, si han interactuado con sus compañeros o con el programa, en qué momento se han publicado los tweets, su relevancia y si contienen o no publicidad.

Tabla 1. Finalistas y semifinalistas de la quinta edición de MasterChef Junior.

\begin{tabular}{|c|c|c|}
\hline Juan Antonio (semifinalista) & 10 años & Twitter: @JuanAntonioMCJ5 \\
\hline Esther (finalista y ganadora) & 10 años & Twitter: @EstherMCJ5 \\
\hline María Arias (semifinalista) & 9 años & Twitter: @MaríaAriasMCJ5 \\
\hline Lucía (finalista) & 12 años & Twitter: @LucíaMCJ5 \\
\hline Gonzalo (finalista) & 12 años & Twitter: @GonzaloMCJ5 \\
\hline
\end{tabular}

Fuente: Elaboración propia

\section{DISCUSIÓN}

Las variables que han sido empleadas para llevar a cabo el análisis de contenido (intención del mensaje, formato, interacción con los compañeros o con el programa, trascendencia del mensaje, contenido publicitario) han aportado cierta lucidez a la hora de observar patrones muy arraigados en cada una de las cuentas que se han analizado. Uno de los puntos de partida del objeto de estudio centraba su mirada en las intenciones publicitarias de MasterChef Junior. Para ello, se ha elaborado una tabla que recoge el porcentaje de mensajes publicitarios presente en los primeros treinta tweets de cada una de las cuentas de los concursantes seleccionados.

Del análisis de los tweets de los cinco aspirantes seleccionados (Esther, Juan Antonio, Lucía, María Arias y Gonzálo) podemos concluir que todos los perfiles mantienen tácticas bastante parecidas y se ha llegado a la deducción de que la mayoría de los mismos se utilizan para promocionar al propio programa. Ya sea mediante retweets o tweets de los propios concursantes, la productora utiliza las redes sociales de los concursantes para hacer llegar a un mayor público aspectos del programa. Lo que más 
se publicita son cada uno de los episodios de MasterChef Junior que se emiten cada semana, aunque también encontramos publicidad acerca de los jueces, invitados y productos del propio programa como cursos de cocina, campamentos... Aunque esto se observa con menor frecuencia.

Sin embargo, no todos los casos son iguales. Por ejemplo, en el Twitter de Juan Antonio se aprecian tendencias que no se ven en los Twitter de los demás concursantes del programa, ya que tiene más tweets dedicados a un uso personal que el resto, aunque esto no quita que siga promocionando la edición a su manera. Este desapego respecto del programa, que no se ve reflejado en el resto de sus compañeros, puede deberse al trato que el resto de concursantes, e incluso, el jurado ha dado a este concursante durante el programa.

Además, esta publicidad no termina con el fin de la edición, sino que continúa una vez que los niños han salido del programa y estos siguen manteniendo las cuentas proporcionadas por MasterChef, lo que sugiere que es probable que exista algún tipo de contrato que les obligue a seguir publicando en sus cuentas tras la finalización del programa.

Gráfico 1. Tweets publicitarios.

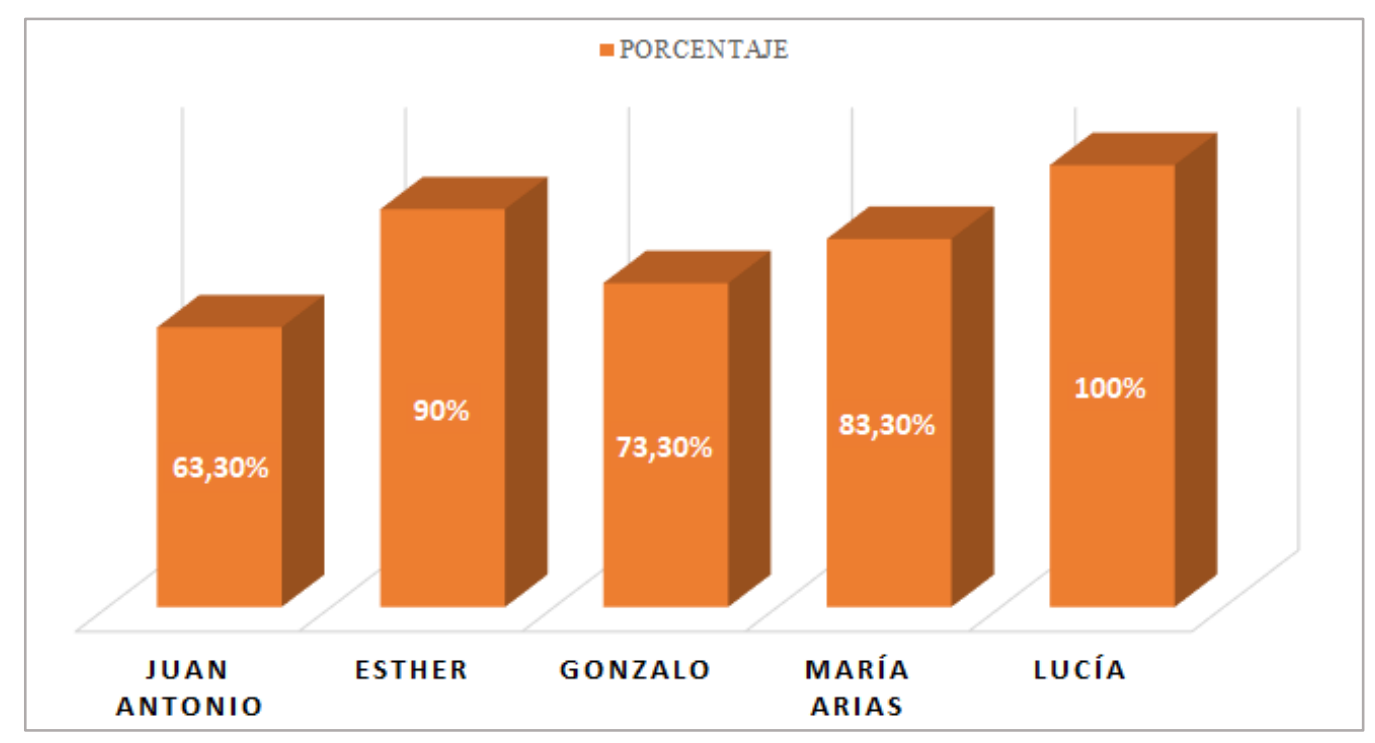

Fuente: Elaboración propia. 
Para llevar a cabo la elaboración de dicha gráfica, se ha tomado en consideración la variable 1 (tweets publicitarios) diseñada para el análisis de contenido. El porcentaje promedio de la publicidad arraigada al total de la muestra examinada es de un 81'98\%, cifra bastante significativa y reveladora a la hora de establecer conclusiones sólidas.

Como podemos observar, cada caso particular posee unas características propias (niños que emplean con poca frecuencia las redes sociales, otros que se muestran muy participativos, algunos que interactúan más con la audiencia, niños que se limitan a compartir contenido solo relacionado con el programa, etc...). No obstante, llama la atención que el participante con menos porcentaje de publicidad en redes sociales (63’30\%) sea aquel que durante el análisis de discurso haya manifestado ciertas actitudes contrarias o distantes a las del resto de sus compañeros, cuyos motivos se explicarán más adelante.

Por otra parte, se contempla un ejemplo bastante contrario en el porcentaje de la participante Lucía (100\%), cuyos treinta primeros tweets están basados en retweets al contenido que publica el programa en redes. Además, se aprecia una trayectoria descendente en el número de publicaciones y el empleo de Twitter a medida que finaliza la edición. Otro dato valioso para esclarecer si la creación de dichas cuentas es o no una parte obligatoria que deriva de la participación en MasterChef Junior.

Gráfico 2. Finalidad de las redes sociales.

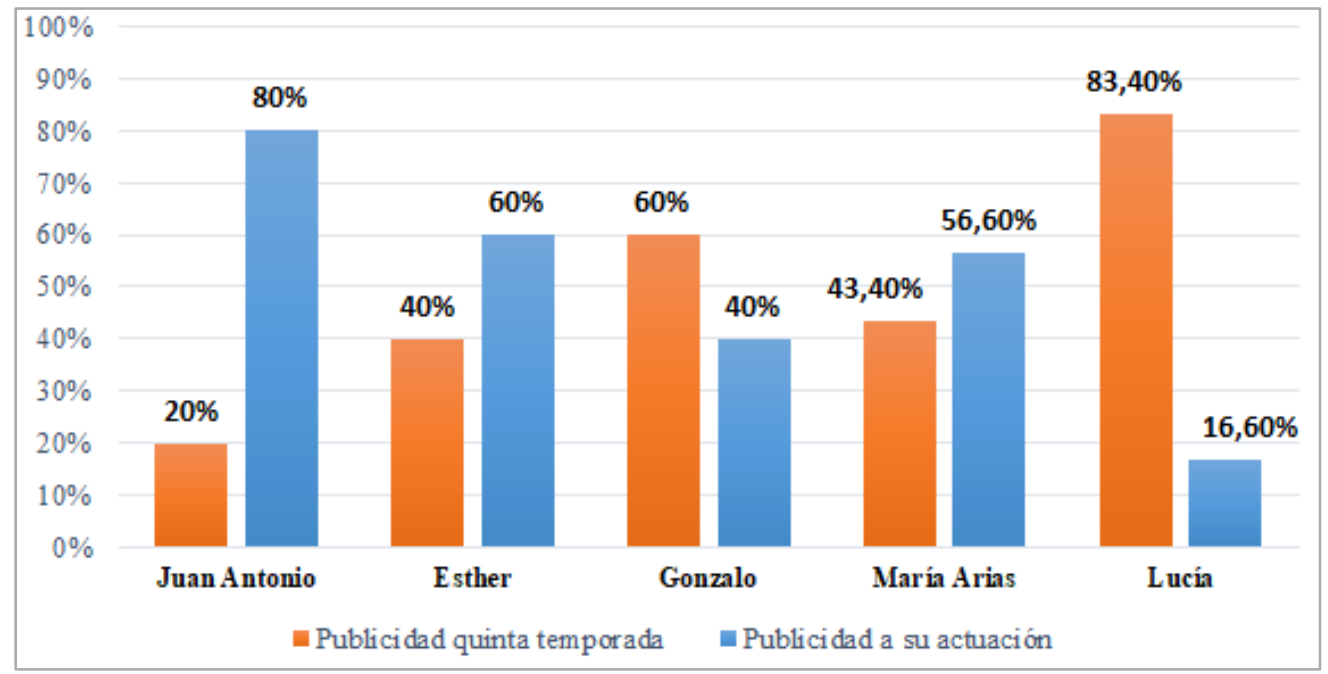

Fuente: Elaboración propia.

RAEIC, Revista de la Asociación Española de Investigación de la Comunicación vol. 7, núm. 13 (2020), 272-289 
El trabajo de campo también ha querido ahondar en otra variable que resulta bastante característica para profundizar en la utilidad que los niños le dan a las redes sociales y que enlaza con la que se ha explicado previamente. Para ello, se ha confeccionado otra tabla que permite dilucidar un poco más las cuestiones en las que se van a profundizar.

Los resultados obtenidos señalan dos posiciones enfrentadas (aquellos que publicitan más al programa o, por el contrario, los que hacen un mayor hincapié en su participación individual). Esto se debe a que las variables examinadas han ido relevando dos perfiles distintos que se advierten con claridad al acudir a las cuentas en las redes sociales. Por ello, los perfiles de los dos niños que anuncian más al programa (es el caso concreto de Lucía y Gonzalo) dejan entrever que la utilización de Twitter se presta más como un deber con el programa que como una herramienta estratégica de cara a la competición, es decir, alcanzar seguidores o fans. Además, esta idea se refuerza con el mínimo uso de la red social por parte de dichos concursantes a medida que culminó la edición.

Sin embargo, la cara opuesta la protagonizan los otros tres concursantes (Juan Antonio, Esther y María Arias). En este caso, la actuación individual es la alternativa que prima en sus redes sociales. No es de extrañar que estos niños posean un mayor porcentaje de actuación individual ya que exteriorizan un perfil muy colaborativo y participativo de cara a la realización de los programas. De ahí, que cuenten con un alto número de seguidores y que protagonicen muchas situaciones que luego son compartidas a través de las redes sociales.

Resulta importante destacar que se han ido realizando ciertos cruces entre los métodos que han sido empleados para la elaboración de este estudio (análisis de discurso y análisis de contenido) y que se explican a continuación:

- Los niños que se muestran menos participativos en redes sociales (Twitter) adquieren un segundo plano en la emisión del programa.

- Aquellos que publicitan en mayor medida su actuación individual se presentan más activos y colaboradores en la realización de las pruebas. 
- En el caso de Juan Antonio, se observa una actitud menos recíproca en redes a la hora de publicitar al programa que los demás, hecho que se refuerza con las valoraciones hacia su persona tanto por parte de los compañeros como de los jueces del concurso.

Adjuntamos dos pruebas estudiadas en el análisis de discurso que ejemplifican la idea que se está defendiendo:

Ilustración 1. Episodio semifinal de MasterChef Junior 5 (0:30:45).

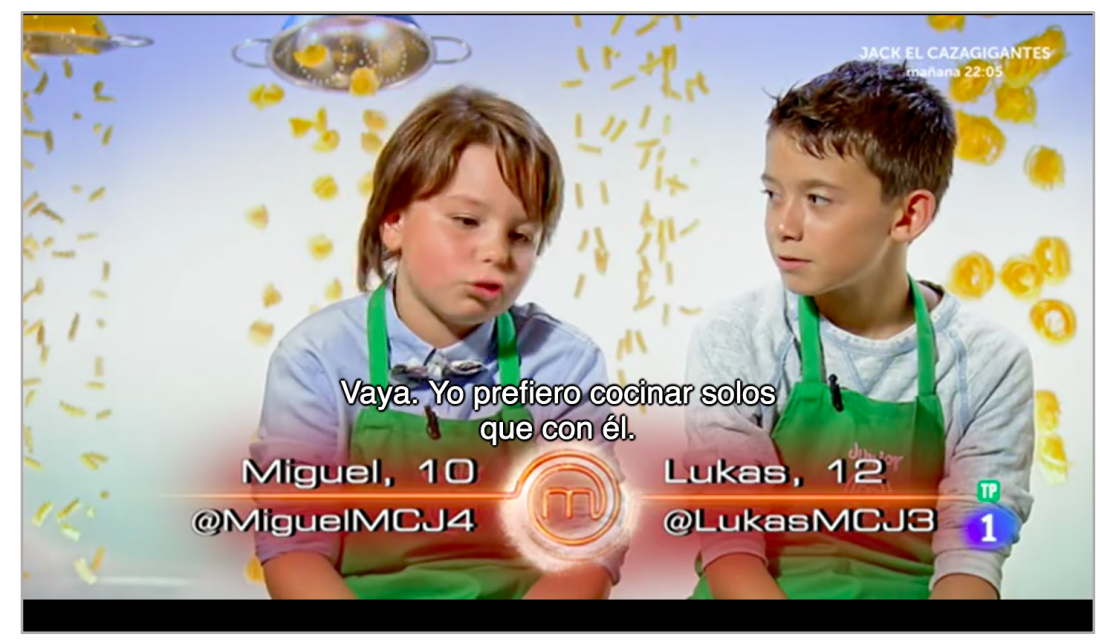

Fuente: RTVE.es.

Sus compañeros hablan en unos totales de que prefieren cocinar solos que con él (con Juan Antonio).

Ilustración 2. Episodio semifinal de MasterChef Junior 5 (0:32:23).

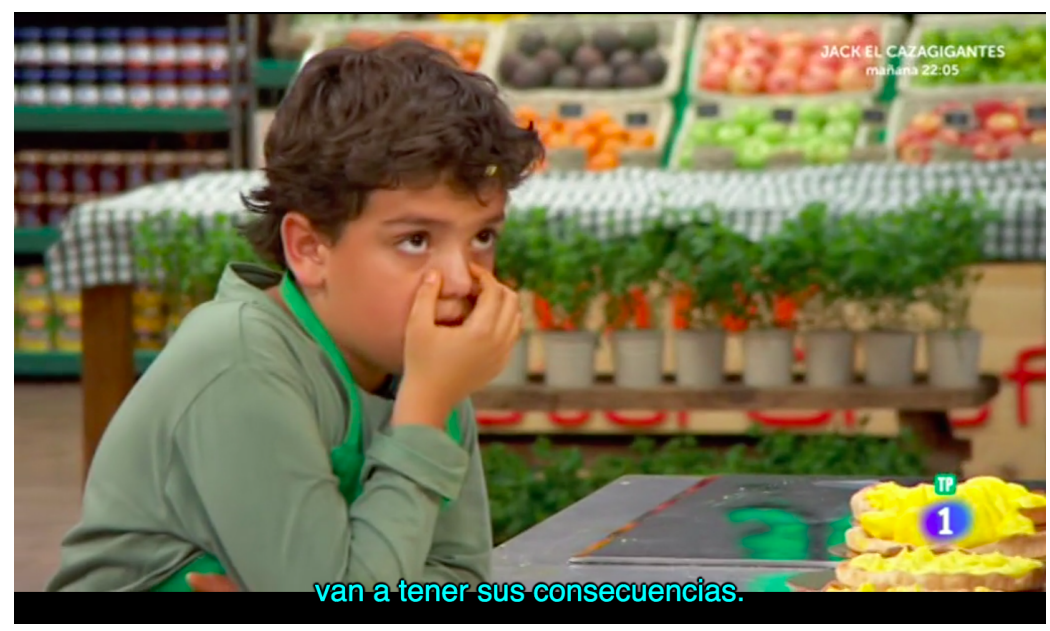

Fuente: RTVE.es.

RAEIC, Revista de la Asociación Española de Investigación de la Comunicación vol. 7, núm. 13 (2020), 272-289 
El juez Jordi Cruz se dirige explícitamente a Juan Antonio tras la realización de una prueba mal hecha y le comenta que va a tener sus consecuencias.

En último lugar, se ha llevado a cabo un análisis de discurso de los dos últimos episodios de la quinta temporada de MasterChef Junior en los que la exigencia con la que se juzga a los concursantes está presente de manera constante durante el visionado de los capítulos.

Se aprecia de manera clara el papel protagonista que adquiere uno de los concursantes, Juan Antonio. En numerosas ocasiones, este participante protagoniza conversaciones solo o en compañía. De igual modo, todos los aspirantes ofrecen totales durante la emisión del episodio y siempre se exhiben las redes sociales de cada uno de ellos como método para integrar a la audiencia, no solo en el visionado sino también en la interacción con el formato a través de otros medios.

Por otra parte, el programa expone un alto nivel de requerimiento que supone un hándicap para los niños que forman parte de la experiencia. En muchas ocasiones, se pueden observar planos en los que los concursantes aparecen llorando, no son capaces de seguir las indicaciones de cocineros profesionales que van a impartir una lección, los jueces deben intervenir para que no desciendan el ritmo e, incluso, circunstancias en las que el jurado se molesta porque una aspirante pregunte a otra algunos de los pasos que se han seguido en las recetas.

Centrándose en el capítulo quinto de la temporada, el cual se corresponde con la semifinal, se pueden observar claras deficiencias del programa en cuanto al tratamiento que se le da a los concursantes, siendo estos niños de entre de entre 9 y 12 años. Se viven situaciones de estrés que influyen en el comportamiento de cara a sus otros compañeros, teniendo espacios aparte en el programa en el que algunos de los participantes se refieren a otros con expresiones como "vago», "desastre» o "prefiero cocinar a solas que con él». Sin embargo, este comportamiento no recibe ninguna sanción por parte de los jueces, sino que, por el contrario, estos emplean repetidamente términos como «la mejor», «el líder», para designar a algunos de los participantes. 
En cuanto a la publicidad que MasterChef introduce en el programa, hay numerosos patrocinios y emplazamientos en los capítulos observados, entre ellos de El Corte Inglés, Bosch y productos propios de MasterChef. Entre los productos propios de Masterchef se encuentra la promoción del campamento, del juego de mesa y de los cursos de cocina online. El patrocinio de Bosch lo anuncian como patrocinio cultural, mientras que el patrocinio de El Corte Inglés no aparece enunciado. No obstante, ambos han sido los patrocinadores oficiales desde la primera edición del programa. Todo esto, teniendo en cuenta que se trata de un programa cuya emisión se realiza en una cadena que desde 2010 no permite la publicidad convencional.

Partimos de la base de que Masterchef no es un programa cultural sino un talent show. Y partimos de esa base porque es la propia descripción que TVE da de ese espacio: «Llega a España MasterChef, el talent show de cocina que ha triunfado en todo el mundo» (Macías, 2013). Puede que la muestra de paisajes españoles sea cultural, o las lecciones gastronómicas, pero ¿hasta qué punto el formato puede considerarse cultural? Resulta cuestionable que un talent show de cocina pueda considerarse el adecuado para un programa cultural (Gallardo, Elías y Jiménez-Marín, 2018). A pesar de las declaraciones que ha hecho Televisión Española para defender el correcto empleo del patrocinio cultural, se puede contemplar como en la web de RTVE. es no se introduce MasterChef en la categoría de programas culturales.

\section{CONCLUSIONES}

Los resultados expuestos en el apartado anterior constatan una serie de estrategias y de usos por parte del programa, MasterChef Junior, y de la productora, Shine Iberia, a través de la redes sociales, haciendo especial hincapié en la red social Twitter. El formato dedica un especial interés a la divulgación de las cuentas de cada uno de los concursantes como forma de conectar más de lleno con la audiencia. Por ello, en todos los totales en los que salen los niños hablando, se muestran los usuarios de cada uno de ellos para dejar patente la idea de una comunidad que va más allá del visionado del episodio y de un trabajo extra que le corresponde a la audiencia de este. 
El interés por alternativas diferentes a la publicidad se debe a que es un programa que se emite en una cadena en la cual no está permitida la misma. Por ello, MasterChef se brinda al empleo de redes sociales que contrarresten el efecto de la ausencia de publicidad y divulgación del formato. Para ello, los aspirantes poseen una cuenta propia que termina con las iniciales del programa y el número de la edición (MCJ5). Esto tiene relación con el análisis de contenido que se ha elaborado acerca de las publicaciones del programa, de la productora y de los concursantes en Twitter y que afirma las intenciones publicitarias que hay en el empleo de estas otras alternativas de difusión.

Esta necesidad de publicitarse a través de las cuentas de los concursantes se debe a que este tipo de formatos ya no son únicamente seguidos a través de la televisión, sino que la audiencia sigue simultáneamente el desarrollo del programa a través de las redes sociales. La audiencia, además, toma un papel activo y no solo lee los posts oficiales de la cadena y concursantes, también interactúa con ellos y crea su propio contenido. De esta forma, actitudes mostradas en el programa son a menudo criticadas por el público en redes sociales. Los propios espectadores a través de redes sociales han denunciado en más de una ocasión la existencia de publicidad encubierta durante la emisión del programa.

Los programas como MasterChef Junior están caracterizados por la importancia dada a la trasmisión de ciertos valores como el compañerismo, el uso de un buen lenguaje o el respeto. En ocasiones el programa traspasa estos valores e integra las características propias que habitualmente contienen los concursos dirigidos a un público adulto, como el sensacionalismo y la competitividad. Ejemplo de ello son las expresiones utilizadas por el jurado a la hora de dirigirse a los niños como «la mejor» o «el líder». Esto hace necesario replantearse si MasterChef Junior es un programa enfocado a espectadores de corta edad o si es necesario que cambie el enfoque hacia una audiencia adulta.

Las principales limitaciones de la investigación provienen de la imposibilidad de contactar con la cadena o productora para obtener información sobre las dinámicas del programa, así como a los propios concursantes a los que se ha tratado de llegar a través de las redes sociales. Respecto a la dificultad para dialogar con los responsables, hay 
que tener en cuenta que se trata de un programa de televisión que se encuentra incluido en un medio de comunicación cuyo principal objetivo es informar a su público. Además, otra dificultad a la hora de realizar la investigación y que podría constituir una futura línea de investigación ha sido discernir si las prácticas publicitarias de MasterChef Junior son publicidad encubierta o legítima, pues según la Ley de Financiación de TVE la cadena no puede emitir publicidad convencional.

\section{REFERENCIAS BIBLIOGRÁFICAS}

Aguilar, M. L. (2015). Proyecto educativo: Masterchef (Trabajo de fin de grado, Universidad de Granada). Recuperado de https://digibug.ugr.es/handle/10481/40638

Alonso, M., Matilla, L. y Vázquez, M. (1995). Teleniños públicos, teleniños privados. Madrid: La Torre.

Ballesta, J. (2002). Educar para el consumo crítico de los medios de comunicación. En Educar en la sociedad digital (pp. 907-916). Granada: Grupo Editorial Universitario, D.L. Comisión Nacional de los Mercados y la Competencia, de 13 de diciembre de 2016. Recuperado de https://www.cnmc.es/sites/default/files/1452099_5.pdf

Digón, P. (2008). Programación infantil y TV sensacionalista: entretener, desinformar y deseducar. Comunicar, 16(31), 65-76.

Encinas, G. (2014). Televisión generalista. El modelo público de TVE frente al privado de Telecinco. (Trabajo fin de grado, Universidad de Extremadura). Recuperado de: http://dehesa.unex.es/bitstream/handle/10662/2147/TFGUEX 2014 Encinas Gil.pdf? sequence $=1 \&$ isAllowed $=y$

Ferrándiz, M. (2014). Gestión de identidad y marca personal en la nueva cocina española. (Trabajo de investigación, Universidad de Alicante). Recuperado de: https://rua.ua.es/dspace/bitstream/10045/38527/1/Gestion de identidad y marca personal FERRANDIZ VANO MARIA DE LOS ANGELES.pdf 
Fuenzalida, V. (2008). Cambios en la relación de los niños con la televisión. Revista Científica de Comunicación y Educación, 5(30), 49-54.

Fuenzalida, V. (2017a). La nueva televisión infantil. Santiago de Chile: Fondo de Cultura Económica.

Fuenzalida, V. et al. (2017b). Nuevos criterios de calidad en la producción de TV infantil. Los desafíos de un canal público para esa audiencia. Whittle J. y Núñez E. (Eds). En VI Panorama del Audiovisual Chileno (pp. 47-62). Santiago de Chile: Dirección de Artes y Cultura de la Pontificia Universidad Católica de Chile.

Gallardo, M., Elías, R. y Jiménez-Marín, G. (2018). La publicidad en Televisión Española tras la ley 8/2009 de Financiación de la Corporación de Radio y Televisión Española. Análisis a través del caso "MasterChef". Pensar la Publicidad, 12, 53-74.

Giordano, E. (2000). Medios de comunicación, ideología y poder. Cuadernos de pedagogía, 297, 28-31.

Livingstone, S. (2007). Los niños en Europa. Evaluación de los riesgos de Internet. Telos: Cuadernos de comunicación e innovación, (73), 52-69.

Livingstone, S. (2015). Active audiences? The debate progresses but is far from resolved. Communication Theory, 25(4), 439-446.

Livingstone, S. (2018). A classe: vivendo e aprendendo na era digital. Comunicação \& Educação, 23(1), 127-139.

Macías, F. (2013): MasterChef, el talent show de cocina que triunfa en todo el mundo. En rtve.es. Recuperado de https://www.rtve.es/television/20130118/tve-estrera-muypronto-masterchef-talent-show-exito-mundial/603710.shtml

Marín, V. (2006). Medios de comunicación, educación y realidad. Comunicar, 26, 193197. 
Mediappro (2006). Mediappro, A European Research Project for the Appropriation of New Media by Youth, Comisión europea. Recuperado de https://www.researchgate.net/publication/282975388 Mediappro The Appropriatio $\underline{\mathrm{n} \text { of New Media by Youth }}$

Moreno, L. (2008). La transmisión de valores en programas infantiles. Comunicar, 16(31), 411-415.

Orozco, G. (1996). Televisión y audiencias: un enfoque cualitativo. Madrid: Universidad Iberoamericana.

Pérez, J.R., y Núñez, L. (2006). Programación infantil en la televisión española. Inadecuada relación entre oferta y demanda. (Trabajo académico, Biblioteca Digital CEU San Pablo). Recuperado de:

https://repositorioinstitucional.ceu.es/bitstream/10637/1200/1/Pag1 11.pdf

Río, M., y Román, M. (Eds.) (2005). Programación infantil de televisión: orientaciones y contenidos prioritarios. Recuperado de:

https://www.observatoriodelainfancia.es/ficherosoia/documentos/1419 d guia infan til IORTV.pdf

Rodríguez, E., Hidalgo, T., y Segarra, J. (2014). La gastronomía como producto de entretenimiento. Análisis de restaurantes y programas culinarios de éxito en España desde una perspectiva creativa. Revista de la Red Académica Iberoamericana de Comunicación, 5(8), 145-157.

Soria, C. (1999). Perspectiva ética en la información publicitaria. En Ética de la comunicación audiovisual (pp. 152-158). Madrid: Tecnos. 